\title{
A novel technique to smoothen harvested rib cartilage for rhinoplasty
}

\begin{abstract}
Use of rib cartilage is widely used for cartilage grafting in rhinoplasty. Although devices are available to smoothen it, they are costly. These include use of cartilage gouze. We suggest a novel method to smoothen the harvested rib cartilage by sandpaper which gives a cheap option with an excellent outcome.
\end{abstract}

Keywords: rhinoplasty, cartilage, grafting, rib, harvested rib cartilage
Volume 9 Issue I - 2017

\section{Shree Harsh, Surendra B Patil,Tarun V \\ Deshbhratar}

Department of Plastic and Maxillofacial Surgery Government

Medical College and Hospital, India

Correspondence: Shree Harsh Department of Plastic and Maxillofacial Surgery Government Medical College and Hospital Nagpur, India,Tel 9I-7028749832, Email s007harsh@gmail.com

Received: August 31, 2017 | Published: November 08, 2017

\section{Introduction}

Rib cartilage for septorhinoplasty for reconstruction of the aesthetic and functional dysfunction of Nose is considered as one of the best replacement options. Restoration of the volume deficit and structural support after primary or secondary rhinoplasty requires rib cartilage. After harvesting the rib cartilage and tailoring it according to the need of Surgery, if there is slight deformity in the contour of the harvested cartilage, it can be evident and will lead to unfavourable outcome after every effort has been made to achieve a picture perfect rhinoplasty.

Various methods have been used to smoothen the rib cartilage. Carving with blade followed by smoothening of the edges with blade is the well known method. Cartilage goose is also described to smoothen the tailored cartilage. We suggest a new method to smoothen out the rough edges of the cartilage.

\section{Materials and methods}

Inclusion criteria- sero negative patients between the age of 18 and 65 years of age with aesthetic deformity of nasal dorsum admitted in Plastic Surgery ward at a tertiary care centre.

\section{Exclusion criteria}

All patients outside inclusion criteria.

\section{Consent}

Written informed consent was taken by the patient and their attendants of a total of 12 patients operated for with aesthetic deformity of nasal dorsum requiring open rhinoplasty admitted in Plastic Surgery department of a tertiary care centre between July 2015 and June 2017, five patients were chosen to undergo smoothening of the harvested rib cartilage with sand paper. All of these 5 patients required dorsal augmentation and application of spreader grafts.

\section{Surgical technique}

After measuring the requirement of the graft, the tailored graft was taken $1 \mathrm{mmm}$ extra in length, width and height.

The tailored cartilage was held in the hand. Autoclaved Number 80 sand paper was wrapped around the handle of a nasal rasp and fixed in place by rubber bands and the rib cartilage graft was rubbed over the surface and edges (Figure 1).

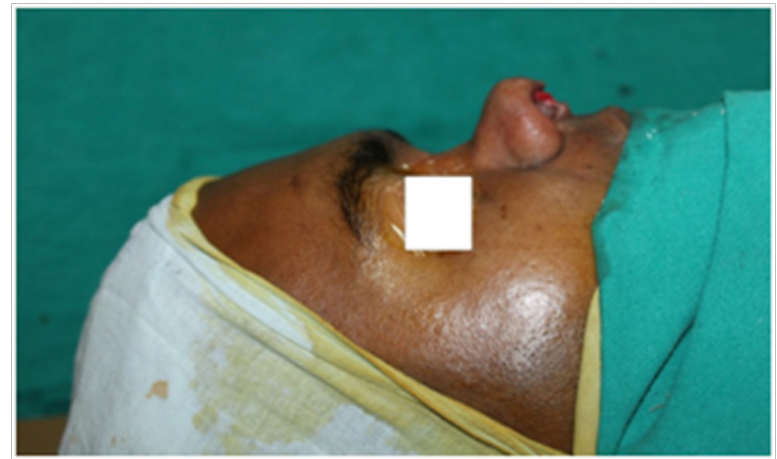

Figure I Preoperative profile view.

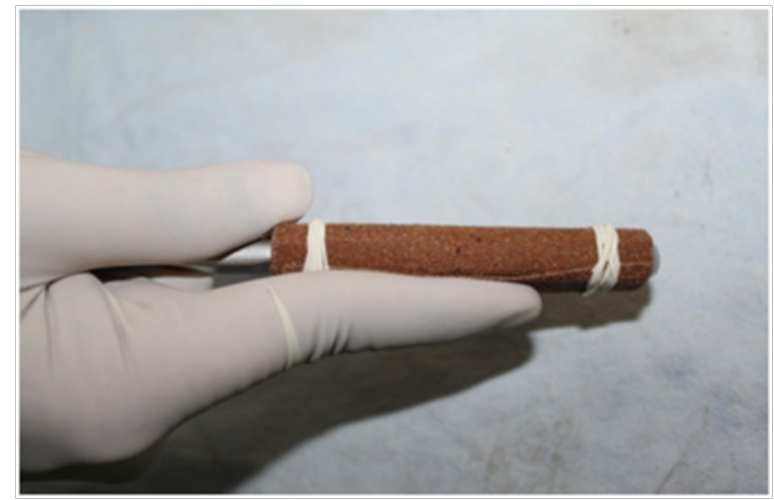

Figure 2 Sandpaper fixed to the gouge.

The surface and edges of the graft were made smooth by repeating this procedure until the desired result was obtained (Figure $2 \& 3$ ). The final smooth rib cartilage graft was washed thoroughly to remove residual sand particles from the graft.

Rest of the procedure adopted was similar to standard open rhinoplasty. Post operative recovery was uneventful and early and late follow-up at 1 year showed excellent aesthetic outcome. Outcomes were seen by examination of the nose by inspection and palpation. 
Postoperative photographs were taken and compared with the preoperative photograph was done.

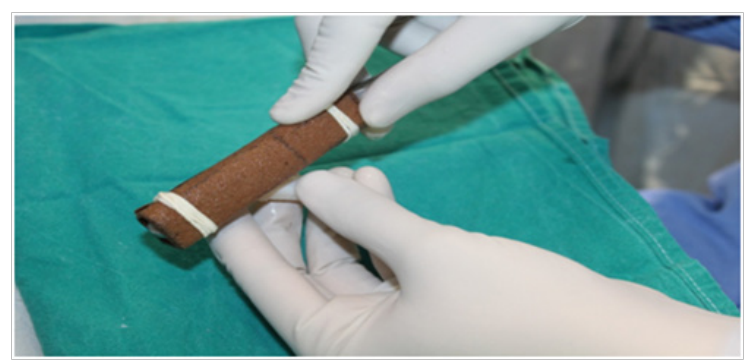

Figure 3 Smoothening of the rib cartilage.

\section{Limitations of the study}

Though the sample size of the study is small, it will increase with the number of cases in future (Figure 4).

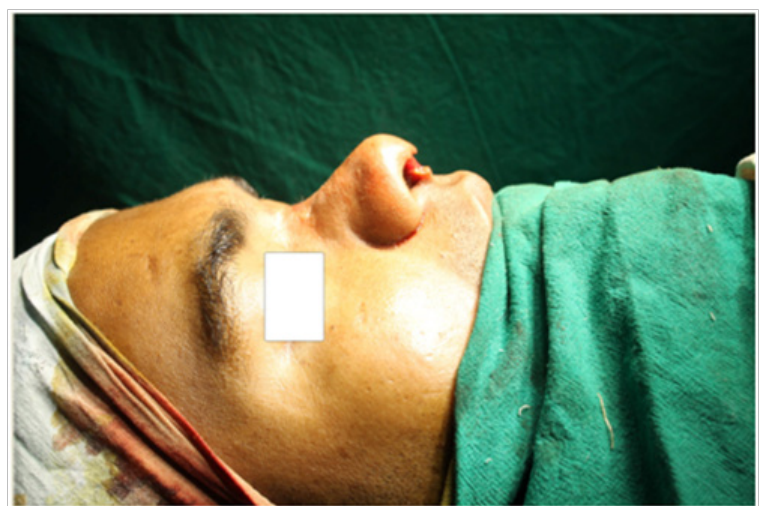

Figure 4 Preoperative photograph.

\section{Discussion}

Carving a costal cartilage can be done by number 10 blade $^{1}$ or numbers, 11 or 15 blades. Once the costal cartilage grafts are fixed in place, any small irregularity on the surface of graft will appear on the skin once edema of the skin subsides especially in thin skinned patients. The edges of the dorsal only graft may show irregularities even after fine trimming. ${ }^{2}$ Dorsal only diced cartilage wrapped in fascia have been used to mask dorsal irregularities. Crushed cartilages have also been used to mask dorsal irregularities. ${ }^{2}$ Glass Polishing disc can be used to smoothen the harvested rib cartilage. ${ }^{3}$ Obtaining a smooth cartilage can also be done by oblique split method. ${ }^{4}$ Smoothing of the harvested costal cartilage with sandpaper has never been described. Proper smoothing will lead to smooth contour resulting in optimal aesthetic outcome. Use of sandpaper is very easy to learn, very easily accessible and cheap.

\section{Conclusion}

Smooth dorsum after primary or secondary rhinoplasty is dependent on the absence of irregularities over the surface of the tailored cartilage. It can result in desired outcome if the surface is devoid of any irregularity. Though some methods have been suggested earlier for the same, we propose a simple, cost effective method to smoothen out the harvested rib cartilage by sand paper.

\section{Conflicts of interest}

Author declares no conflict of interest.

\section{Acknowledgments}

None.

\section{Funding}

None.

\section{References}

1. Eelam A Adil. The harvest of costal cartilage in Rhinoplasty. 2011.

2. Ozcan Cakmak, Fuat Buyuklu. Crushed cartilage grafts for concealing irregularities in rhinoplasty. Arch Facial Plast Surg. 2007;9(5):352-357.

3. Agarwal K, Shrotria R. Ingenious technique of smoothening costal cartilage graft by glass polishing disc. Indian J Plast Surg . 2016;49(2):288-299.

4. Tastan E, Yucel OT, Adyin E, et al. The oblique split method: a novel technique for carving costal cartilage grafts. JAMA Facial Plast Surg. 2013;15(3):198-203. 